\title{
Game Theoretic Efficient Radio Resource Allocation in 5G Resilient Networks: A Data Driven Approach
}

\author{
Ahmad Mudassir*1 | Syed Ali Hassan² | Haris Pervaiz ${ }^{3}$ | Saleem Akhtar ${ }^{1}$ | Hesham Kamel ${ }^{4}$ | Rahim \\ Tafazolli $^{3}$
}

${ }^{1}$ Electrical Engineering, Comsats University Islamabad, Lahore campus, Lahore, Pakistan

${ }^{2}$ School of Electrical Engineering and Computer Science (SEECS), National University of Sciences and Technology (NUST), Islamabad, Pakistan

${ }^{3}$ ICS, Home of 5GIC, University of Surrey, Guildford, UK

${ }^{4}$ Electrical Engineering, Egyptian Chinese University, Cairo, Egypt

Correspondence

*Ahmad Mudassir, Comsats University

Islamabad, Lahore Campus. Email:

amudassir@ciitlahore.edu.pk

\begin{abstract}
Summary
In recent years, $5 \mathrm{G}$ resilient networks have gained significant attention in the wireless industry. The prime concern of commercial networks is to maximize network capacity to increase their revenue. However, in disaster situations during outages when cell sites are down, instead of capacity, coverage becomes predominant. In this paper, we propose a game theory-based optimal resource allocation scheme, while aiming to maximize the sum-rate and coverage probability for the uplink transmissions in disaster situations. The proposed hierarchical game theoretical framework optimizes the uplink performance in three-tier heterogeneous network with pico base stations, femto access points overlaid under macro base station. The test simulations are based on a real time data set obtained for a pre-defined amount of time. The data statistics are then manipulated to create practical disaster situations. The solution for the noncooperative game has been obtained by using pure strategy Nash equilibrium. We perform simulations with different failure rates and the results show that proposed scheme improves the sum rate and outage probability by significant margin with or without disaster scenario.
\end{abstract}

\section{1 | INTRODUCTION}

Because of the recent exponential growth in the data traffic, bigger data rates have become the essential requirement for next generation mobile networks. However, mobile operators are facing decoupling of their revenue and network traffic due to regulatory pressure, flat-rate tariffs, competitive and saturated markets ${ }^{1}$. To meet this demand and increase the network efficiency, 3GPP specified dense small cells-based heterogeneous networks (HetNets), which have emerged as a powerful domain for research and development. A multi-tier HetNet comprises of small power pico base stations (BSs) with slightly greater transmit power than femto BSs overlaid under a high power macro BS. A considerable literature has appeared recently in the domain of HetNets to address the challenges related to radio resource management, network modeling and energy efficiency ${ }^{23 / 4}$, to name a few.

The major concerns in a multi-tier HetNet is severe intercell interference in co-channel deployment, user association and resource allocation ${ }^{516}$ etc. Cross tier interference is one of the greatest challenges in multi-tier HetNets and it has received considerable attention. Existing research recognizes the role played by interference management and sum rate enhancement to improve the system performance ${ }^{7}$. HetNets are different from classical wireless networks because of the increased number of configurations with the deployed small base stations. Therefore, the massive overhead in computation and signaling associated with HetNets cannot be handled by existing centralized resource management algorithms as in ${ }^{899}$. Also competing interests of users and base stations require coordination and tradeoff. Hence to enable small cell network to operate with low overhead, distributed self-organizing algorithms must be 
designed 10. Self-organization allows small cells to achieve optimal performance by learning from their environment and updating their configuration policies without external control.

The salient components of HetNets include Femto Access Points (FAPs) and Pico Base Stations (PBSs). FAPs are typically designed to use in a home or small business and can operate in open, close or hybrid access modes. In an open access mode, FAPs distribute their resources among home users and macrocell users to handle the interference and to boost the overall sum-rate. Whereas in closed access mode, a FAP dedicates all its resources to its home users. PBSs are small cellular base station typically providing coverage and capacity in a small indoor/outdoor area, such as in shopping malls and train stations. The PBSs offload traffic from MBS to improve coverage probability and reduce outage events.

In this paper, we have proposed a game theoretical approach in multi-tier HetNets to sustain coverage, improve capacity and resilience in case of disaster and network failures. Game theory deals with the distributed optimization wherein the selfish users can make their own rational decisions, instead of being controlled by central authority. Because of distributed nature, the system designs based on the game-theoretical approach are expected to be highly scalable.

To optimize the sum-rate of a heterogeneous network, our tiered game theory-based design comprising of two sub-games for resource allocation. In the first game, the approach starts by favored access policies of FAPs and to fractionate the competing interests of FAPs and PBSs. In the second game, while taking into account the network performance, MUEs finalize their association to FAPs and PBSs through a user-centric scheme in the interest of enhancing their sum-rate. This distributed scheme perpetually accomplishes a pure strategy Nash equilibrium (PSNE).

The main contributions of this work are summarized as follows:

- We propose a distributed user association scheme for three-tier HetNet to investigate the sum-rate maximization subject to the minimal outage probability (or maximum coverage probability) and minimum QoS requirements to dynamically allocate the bandwidth among the different set of users in comparison to the traditional all-closed scheme.

- The proposed scheme is evaluated to investigate the network resilience in disaster and network failure situations.

- We have simulated the disaster and network failure scenarios in order to evaluate the system performance in terms of coverage and outage probabilities on different traffic loads by varying the minimum Quality of Service (QoS) requirements.
- We have tested our design through rigorous simulations and showed that at higher failure rate our model is providing better coverage even at higher QoS requirements and at high traffic load which is rarely seen in the literature.

The rest of the paper is organized as follows, Section II describes the current state-of-the-art relevant to the multitier HetNet. We then describe the system model followed by the formulation of the optimization problem and the proposed solution to solve the formulated problem as described in Section III. Finally, Section IV discusses simulation results followed by the conclusion in Section V.

\section{2 | RELATED WORK}

Game theoretical approaches have gained significant attention in recent research to develop self organization algorithms for HetNet to analyze resource allocation, power control, interference mitigation and user association.

Decentralized and self organizing mechanism for interference management in closed access and small cells with minimum information required to learn an equilibrium is proposed in 111 . In this algorithm, the femto BS relies solely on local information in the form of feedback by jointly estimating their long-term utilities and optimizing their probability distribution of choosing their strategies, while adhering to the cross-tier interference constraint set by macro cell. The classes of games which includes, identical interest, potential and weekly acyclic with the perspective of log-linear learning are discussed in 12 . In each of these class of games, a pure Nash equilibrium is guaranteed to exist wherein the players are aware of received utility and player actions whereas the structural requirements of the log-linear learning are relaxed.

The authors in ${ }^{[13}$ modeled network selection problem as non-cooperative evolutinary game among multiple groups of users (players) in heterogeneous wireless network and Nash equilibrium was obtained as the solution of the game. 14 proposed a cooperative solution that enables femtocells to improve the achievable data rates by suppressing intra-tier interference using the concept of interference alignment. This cooperative behavior is modeled among the femtocells as coalition game in a partition form. Coalition game in partition form between femto BS through distributed solution allows them to autonomously form coalition to increase their revenue (i.e, their total rate), given the cost of co-operation is captured by the utility function. The authors in ${ }^{[15}$ designed a distributed mechanism that jointly performs associating the UEs to APs and allocates femto BSs to the service providers, such that the total satisfaction of the UEs in an uplink Orthogonal Frequency Division Multiple Access (OFDMA) network is maximized. 
Co-operative behavior amongh the UEs, FBSs and Service Providers (SPs) is modeled as dynamic matching game to find the optimal UE association and FBS allocation. The authors in 16 developed cooperative interference management model for small cell network in which small base stations are able to participate and cooperate to optimize their sum rate with multiple overlapping coalitions. A co-channel assignment approach is adopted that improves the spectral efficiency compared to the orthogonal spectrum allocation. It was shown that the proposed game has a transferable utility and exhibits negative externalities due to the co-tier interference between small cell base stations.Authors proposed a Nash bargaining based power efficient resource control scheme for cognitive radio systems which is outlined in 17 .

The technical specifications of a $5 \mathrm{G}$ resilient networks have not yet been standardized but such networks are needed to provide a high level of reliability and resilience to failures in order to guarantee the availability to the customers such as common public and public safety responders when needed. One of the works in the recent literature ${ }^{18}$ has investigated the resiliency of cellular base station deployment through a measurement method in conjunction with a performance metric such as coverage or supported throughput.

According to the best of our knowledge, there has been no detailed investigation on interference avoidance and sum rate improvement in multi-tier HetNets specially from the perspective of $5 \mathrm{G}$ resilient networks.

\section{3 | SYSTEM MODEL AND ASSUMPTIONS}

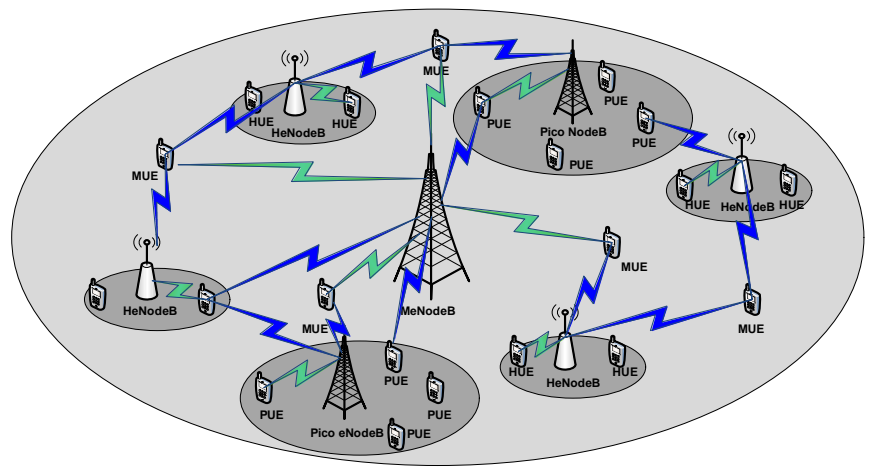

FIGURE 1 Fully Functional Multi-Tier Heterogeneous Network Deployment Scenario.

A multi-tier heterogeneous network scenario in the uplink transmission scheme is considered, having $N F A P S$ and $P$ pico base stations overlaid within a macro cell, as shown in Figure 1. There are $M$ macrocell user equipments (MUEs). Let $\mathbb{N}=$

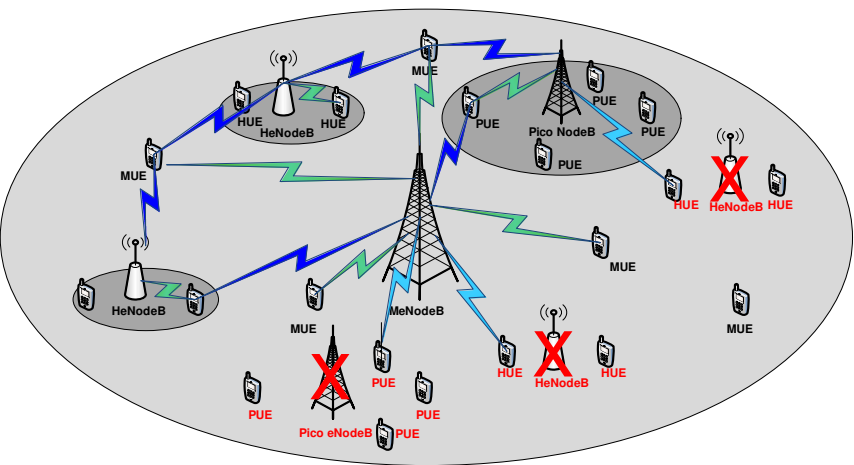

FIGURE 2 Multi-Tier Heterogeneous Network Deployment Scenario with Infrastructure Failure in the Disaster Region.

$\{1,2, \ldots, N\}$ be the set of FAPs, $\mathbb{P}=\{1,2, \ldots, P\}$ be the set of PBSs, $\mathbb{M}=\{1,2, \ldots, M\}$ be the set of MUEs, $\mathbb{A}=\{1,2, \ldots, A\}$ be the set of femtocell users and $\mathbb{B}=\{1,2, \ldots, B\}$ be the set of picocell users, The division of system bandwidth $B W$, is done among FAPs such that $B W_{\mathrm{FAP}}=\frac{B W}{|\mathbb{N}|}$ and each FAP has $K$ subcarriers available, wherein the bandwidth of each subcarrier is given by $\frac{B W_{\mathrm{FAP}}}{K}$. In this way FUEs do not create co-tier interference on the uplink. But FUEs, will create cross tier interference with PBSs and MBS. Similarly system bandwidth, is equally segregated with PBSs such as $B W_{\mathrm{PBS}}=\frac{B W}{|\mathbb{P}|}$ and each PBS has $C$ subcarriers available, wherein each subcarrier has the available bandwidth given by $\frac{B W_{\mathrm{PBS}}}{C}$. This assures that the PUEs do not create co-tier interference on the uplink as different PBSs are assigned orthogonal bands using OFDMA. But PUEs will create cross tier interference with FAPs and MBS. The macro base station $(M B S)$ share the same bandwidth $B W$ with FAPs and PBSs, which is segregated equally among the $L$ subcarriers such that the available bandwidth of each subcarrier is given by $\frac{B W}{L}$. In this way, MUEs will introduce cross-tier interference to the FAPs and PBSs. The deployment scenario for the multi-tier HetNet is depicted in Figure 1.

\section{1 | Data Driven Networking Approach Towards 5G Resilient Networks}

In case of the disaster or network failure situations, the conventional systems do not provide sufficient communication facilities for relief and rescue operations. In this situation, damages in the infrastructure may leave many people isolated. For the $5 \mathrm{G}$ resilient networks, it is very important to compensate outage and recover network communication independently during disasters or network failure. As shown in Figure 2 that in case of the network failure, the users are required to be re-associated with the existing and functional network infrastructure utilizing the game theoretical concepts. 
The historical data available about the network infrastructures can be utilized to get an average predicted traffic loads for the specific geographical region at the different times of a specific day of the week which is outlined in 19220 . This historical data available can be utilized along with the game theoretical concepts to accurately predict the optimal number of network infrastructure needed to provide the sufficient QoS without any degradation. The availability of this abundant historical data about the network infrastructures can pave the way towards the data driven networking to enable the intelligence and adaptability in the future generation networks towards self-organizing and self-healing $5 \mathrm{G}$ resilient networks. The historical data about the network infrastructures can be measured at different times of the days, i.e., $15 \mathrm{~min}$ or $30 \mathrm{~min}$ intervals. The whole day historical data will compromise of 48 (or 96) measurements depending on the measuring intervals of $30 \mathrm{~min}$ (or $15 \mathrm{~min}$ ).

The traffic patterns such as peak and off peak times are quite dependent on both time and day of the week. The historical data can be split into two different subsets, i.e., training and validating data. The machine learning concepts such as support vector machine (SVM) regression model can be applied on the training data to train the system. The accuracy of the system is quite dependent on the size of the training data to overcome the affect of Outliers (usually set at 3 times the standard deviation) and also the missing measurement values in the historical training data. Once the system is trained, then the system is evaluated on the validating data in order to measure the effectiveness of the trained system. Lets us define the actual average traffic load at the time $i$ of the day $j$ by $t_{i, j}$, wherein $i \in$ $\{1,2,3, \cdots, Y\}, j \in\{$ Mon, Tue, Wed,Thu, Fri, Sat, Sun $\}$ and $Y$ depends on the measuring interval given by $Y=$ $\frac{24}{m_{\text {interval }}}$. It is important to highlight that mostly $m_{\text {interval }}$ is between 5 to 30 mins and smaller the $m_{\text {interval }}$, the better is the efficiency and accuracy. Based on our proposed machine learning approach, we can predict the average traffic load at the time $i$ of the day $j$ by $\tilde{t}_{i, j}$. The efficiency of the proposed system can be measured as follow:

$$
\Delta t_{i, j}=\frac{\left|t_{i, j}-\tilde{t}_{i, j}\right|}{t_{i, j}}
$$

For the every predicted average traffic load $\tilde{t}_{i, j}$ at the given time $i$ of the day $j$ in the disaster hit region from the historical traffic data, the optimization problem is formulated to maximize the system sum rate subject to the minimum outage probability and minimum QoS requirement constraints. The formulated problem and the proposed distributed noncooperative game theoretic solution are described in detail in Section 3.2 and 3.3, respectively.
Lets us also define the two key terms namely as traffic load and network failure rate at the time $i$ of the day $\sqrt{1}$ used throughout the paper as follow:

Traffic Load $[\%]=\left(M+|\mathbb{N}| \times U_{\mathbb{N}}+|\mathbb{P}| \times U_{\mathbb{P}}\right) \times \frac{t_{i, j}}{100}$,

Failure Rate[\%] $=\frac{\left(1+\left(|\mathbb{N}|-A_{\mathbb{N}} \times|\mathbb{N}|\right)+\left(|\mathbb{P}|-A_{\mathbb{P}} \times|\mathbb{P}|\right)\right)}{(1+|\mathbb{N}|+|\mathbb{P}|)}$

where $U_{\mathbb{N}}$ denotes the number of FUEs per FAP, $U_{\mathbb{P}}$ denotes the number of PUEs per PBS, $A_{\mathbb{N}}$ denotes the proportion of FAPs that are fully functional and $A_{\mathbb{P}}$ denotes the proportion of PBSs that are fully functional.

\section{2 | Problem Formulation}

The signal to interference-plus-noise ratio (SINR) for the $a^{\text {th }}$ FUE at $n^{\text {th }}$ FAP is given by

$$
\Gamma_{a}^{n}[k]=\frac{\mu_{a}^{n}[k]}{\sigma^{2}[k]+I_{m}^{n}[k]+I_{p}^{n}[k]}
$$

where $\mu_{a}^{n}[k]=\left(h_{a}^{n}[k]\right)^{2} P_{a} W\left(d_{a}^{n}\right)^{-\beta}$ is the received power from $a_{t h}$ FUE at $n_{t h}$ FAP on $k_{t h}$ subcarrier, $h_{a}^{n}[k]$ is the channel fading, $d_{a}^{n}$ is the distance, $P_{a}$ is the FUE transmit power and $W$ is the wall penetration loss. The denominator terms are given as; $\sigma^{2}[k]$ is the noise variance at $k^{\text {th }}$ subcarrier.

$$
I_{m}^{n}[k]=\sum_{m=1}^{M}\left(\prod_{n=1}^{N} \rho_{m}^{n}[k]=0\right) \mu_{m}^{n}[k]
$$

where $\rho_{m}^{n}[k] \epsilon\{0,1\}$ signifies the connection between $m_{t h}$ MUE to $n_{t h}$ FAP at $k_{t h}$ subcarrier.

$$
I_{p}^{n}[k]=\sum_{p=1}^{P} \sum_{b=1}^{B} \mu_{b, p}^{n}[k]
$$

The signal to interference-plus-noise ratio (SINR) for the $m^{\text {th }}$ MUE at $n^{\text {th }}$ FAP is given by

$$
\Gamma_{m}^{n}[k]=\frac{\left[1-\left(\prod_{n=1}^{N} \rho_{m}^{n}[k]=0\right)\right] \mu_{m}^{n}[k]}{\sigma^{2}[k]+I_{m}^{n}[k]+I_{p}^{n}[k]}
$$

where $\mu_{m}^{n}[k]=\left(h_{m}^{n}[k]\right)^{2} P_{m}\left(d_{m}^{n}\right)^{-\alpha}$ is the received power from $m_{t h}$ MUE at $n_{t h}$ FAP on $k_{t h}$ subcarrier, $h_{m}^{n}[k]$ is the channel fading, $d_{m}^{n}$ is the distance and $P_{m}$ is the MUE transmit power.

The signal to interference-plus-noise ratio (SINR) for the $b^{\text {th }}$ PUE at $p^{\text {th }}$ PBS is given by

$$
\Gamma_{b}^{p}[c]=\frac{\mu_{b}^{p}[c]}{\sigma^{2}[c]+I_{m}^{p}[c]+I_{n}^{p}[c]}
$$

\footnotetext{
${ }^{1}$ the subscript $i$ and $j$ are dropped throughout the remainder of the paper from the purpose of simplicity and ease.
} 
where $\mu_{b}^{p}[c]=\left(h_{b}^{p}[c]\right)^{2} P_{b}\left(d_{b}^{p}\right)^{-\alpha}$ is the received power from $b_{t h}$ PUE at $p_{t h}$ PBS on $c_{t h}$ subcarrier, $h_{b}^{p}[c]$ is the channel fading, $d_{b}^{p}$ is the distance and $P_{b}$ is the PUE transmit power. where

$$
\begin{gathered}
I_{m}^{p}[c]=\sum_{m=1}^{M}\left(\prod_{p=1}^{P} \rho_{m}^{p}[c]=0\right) \mu_{m}^{p}[c] \\
I_{n}^{p}[c]=\sum_{n=1}^{N} \sum_{a=1}^{A} \mu_{a, n}^{p}[c]
\end{gathered}
$$

The signal to interference-plus-noise ratio (SINR) for the $m^{\text {th }}$ MUE at $p^{\text {th }} \mathrm{PBS}$ is given by

$$
\Gamma_{p}^{m}[c]=\frac{\left[1-\left(\prod_{p=1}^{P} \rho_{m}^{p}[c]=0\right)\right] \mu_{m}^{p}[c]}{\sigma^{2}[c]+I_{m}^{p}[c]+I_{n}^{p}[c]}
$$

The signal to interference-plus-noise ratio (SINR) for the $m^{\text {th }}$ MUE at MBS is expressed as

$$
\Gamma_{m}^{o}[l]=\frac{\left(\prod_{n=1}^{N} \rho_{m}^{n}[l]=0\right)\left(\prod_{p=1}^{P} \rho_{m}^{p}[l]=0\right) \mu_{m}^{o}[l]}{\sigma^{2}[l]+I_{m}^{o}+I_{n}^{o}+I_{p}^{o}}
$$

where

$I_{m}^{o}=\left[1-\left(\prod_{n=1}^{N} \rho_{m}^{n}[l]=0\right)\right]\left[1-\left(\prod_{p=1}^{P} \rho_{m}^{p}[l]=0\right)\right] \mu_{m}^{o}[l]$

where

$$
\begin{aligned}
& I_{n}^{o}=\sum_{n=1}^{N} \sum_{a=1}^{A} \mu_{a, n}^{o}[l] \\
& I_{p}^{o}=\sum_{p=1}^{P} \sum_{b=1}^{B} \mu_{b, p}^{o}[l]
\end{aligned}
$$

The FAP strategy vector consists of the portion of frequency band distributed to each MUE and the rate of its FUEs is utility function, which can be represented as

$\zeta_{n}\left(\varkappa_{n}, \varkappa_{-n}\right)=\sum_{k=1}^{K}\left(\prod_{n=1}^{N} \rho_{m}^{n}[k]=0\right) \log \left(1+\sum_{n=1}^{N} \sum_{a=1}^{A} \Gamma_{a}^{n}[k]\right)$ where $\varkappa_{n}=\left[\varkappa_{1, n}[1], \ldots, \varkappa_{M, n}[1], \varkappa_{1, n}[2], \ldots, \varkappa_{M, n}[K]\right]^{T}$ is the strategy vector of $n_{t h}$ FAP. where $\varkappa_{-n}=$ $\left[\varkappa_{1}^{T}, \ldots, \varkappa_{n-1}^{T}, \varkappa_{n+1}^{T}, \ldots, \varkappa_{N}^{T}\right]^{T}$ is the strategy vector of other FAPs.

The FAPs, PBS and MBS allocated the portion of band to MUEs which become their strategy vectors the utilities are their rates. The utility function can be formulated as

$$
\begin{array}{r}
\zeta_{m}\left(\varkappa_{m}, \varkappa_{-m}\right)= \\
\sum_{k=1}^{K}\left[1-\left(\prod_{n=1}^{N} \rho_{m}^{n}[k]=0\right)\right] \log \left(1+\sum_{m=1}^{M} \Gamma_{m}^{n}[k]\right)+ \\
\sum_{l=1}^{L}\left[\left(\prod_{n=1}^{N} \rho_{m}^{n}[l]=0\right)\right] \log \left(1+\Gamma_{m}^{o}[l]\right)+ \\
\sum_{c=1}^{c}\left[\left(\prod_{p=1}^{P} \rho_{m}^{p}[c]=0\right)\right] \log \left(1+\Gamma_{m}^{o}[c]\right)
\end{array}
$$

where $\varkappa_{m}=\left[\varkappa_{1, m}[1], \ldots, \varkappa_{N, m}[1], \varkappa_{1, m}[2], \ldots, \varkappa_{N, m}[K]\right.$, $\varkappa_{1, m}[1], \ldots, \varkappa_{N, m}[1], \varkappa_{1, m}[2], \ldots, \varkappa_{N, m}[K]^{T}$ is the strategy vector of $m_{t h}$ MUE and where $\varkappa_{-m}=$ $\left[\varkappa_{1}^{T}, \ldots, \varkappa_{n-1}^{T}, \varkappa_{n+1}^{T}, \ldots, \varkappa_{N}^{T}\right]^{T}$ is the strategy vector of other MUEs.

The PBS strategy vector consists of the portion of frequency band distributed to each MUE and the rate of its PUEs is utility function, which can be defined as

$\zeta_{p}\left(\varkappa_{p}, \varkappa_{-p}\right)=\sum_{c=1}^{C}\left(\prod_{p=1}^{P} \rho_{m}^{p}[c]=0\right) \log \left(1+\sum_{p=1}^{P} \sum_{b=1}^{B} \Gamma_{b}^{p}[c]\right)$

where $\varkappa_{p}=\left[\varkappa_{1, p}[1], \ldots, \varkappa_{M, p}[1], \varkappa_{1, p}[2], \ldots, \varkappa_{M, p}[C]\right]^{T}$ is the strategy vector of $p_{t h}$ PBS. where $\varkappa_{-p}=$ $\left[\varkappa_{1}^{T}, \ldots, \varkappa_{p-1}^{T}, \varkappa_{p+1}^{T}, \ldots, \varkappa_{P}^{T}\right]^{T}$ is the strategy vector of other PBSs.

The minimum acceptable rate for MUE is $\gamma_{\min }$ which is fixed for all MUEs in the network. The connectivity constraint between $n_{t h}$ FAP and $m_{t h}$ MUE, is represented by

$$
\left(1-\prod_{n=1}^{N} \rho_{m}^{n}[k]=0\right) \gamma_{\min } \leq \sum_{k=1}^{K} \rho_{m}^{n}[k] \log \left(1+\Gamma_{m}^{n}[k]\right)
$$

The minimum acceptable data rate constraint for connectivity between $m_{t h}$ MUE and MBS, this constraint is given by

$$
\left(\prod_{n=1}^{N} \rho_{m}^{n}[l]=0\right) \gamma_{\min } \leq \sum_{l=1}^{L} \rho_{n}^{o}[l] \log \left(1+\Gamma_{m}^{o}[l]\right)
$$

The minimum acceptable data rate for connectivity between $m_{t h} \mathrm{MUE}$ and $p_{t h} \mathrm{PBS}$, this constraint is given by

$$
\left(1-\prod_{p=1}^{P} \rho_{m}^{p}[c]=0\right) \gamma_{\min } \leq \sum_{c=1}^{C} \rho_{m}^{p}[c] \log \left(1+\Gamma_{m}^{p}[c]\right)
$$

In the first game the strategy space for $n_{t h}$ FAP and $p^{t h}$ PBS is given by equation (19) and (20) respectively;

$$
\begin{aligned}
& \tilde{\psi}_{n}=\left\{\rho_{m}[k] \epsilon(0,1)^{M K}: \sum_{m=1}^{M} \rho_{m}^{n}[k] \leq 1\right\} \\
& \tilde{\psi}_{p}=\left\{\rho_{m}[c] \epsilon(0,1)^{M C}: \sum_{m=1}^{M} \rho_{m}^{p}[c] \leq 1\right\}
\end{aligned}
$$

The constraint shown above ensures that only one MUE can connect to $n_{t h}$ FAP on $k_{t h}$ subcarrier and $p_{t h}$ PBS on $c_{t h}$ subcarrier respectively. On the basis of given strategy vectors of other FAPs and PBSs the solution of optimization problem by $n_{t h}$ FAP and $p_{t h}$ PBS can be defined as;

$$
\begin{aligned}
& \max _{\rho_{n} \in \tilde{\psi_{n}}}\left(\rho_{n}, \rho_{-n}\right) \\
& \max _{\rho_{p} \epsilon \tilde{\psi_{p}}}\left(\rho_{p}, \rho_{-p}\right)
\end{aligned}
$$


For second game the strategy space for $m_{t h}$ MUE can expressed as;

$$
\widetilde{\psi_{m}}=\left\{\rho_{m}[l] \epsilon(0,1)^{(N+1) L}:\left(\rho_{m}^{n}[l]+\rho_{m}^{p}[l]+\rho_{m}^{b}[l]\right) \leq 1\right\}
$$

The above equation applies the constraint not to associate MUE to FAP, PBS and MBS simultaneously.

$$
\max _{\rho_{m} \in \tilde{\psi}_{n}}\left(\rho_{n}, \rho_{-n}\right)
$$

The above games are solved by Nash Equilibrium, which is attained by $\left(x_{i}^{*}, x_{-i}^{*}\right)$ when

$$
\tilde{f}_{i}\left(x_{i}^{*}, x_{-i}^{*}\right) \geq \tilde{f}_{i}\left(x_{i}^{*}, x_{-i}^{*}\right) \forall x_{i} \in \tilde{\psi}_{i}
$$

\section{3 | Proposed User-Centric Multi-Tier Resource Allocation Scheme: A Hierarchical Game Theoretic Approach}

A distributed user-centric solution is proposed with emphasis on maximizing the data rate offered to the users by optimizing the resolution between interference and resources. While achieving stable action profiles, the algorithm always reaches to pure strategy Nash equilibrium. The algorithm allows FAPs and PBSs to select their strategies in the $i^{\text {th }}$ iteration keeping in view the strategies of other FAPs and PBSs respectively in the $(i-1)^{\text {th }}$ iteration. This is done at any point in time using a parallel update technique. In the start without seeking an equilibrium, optimal resources are allocated to all MUEs by constructing the initial strategy vectors for FAPs and PBS while satisfying minimum data rate requirement using.

$$
\begin{aligned}
\omega_{m}{ }^{n} & =\frac{\gamma_{\min }}{\log _{2}\left(1+\frac{\Gamma_{m}^{n}}{\sigma^{2}}\right)} \\
\omega_{m}{ }^{p} & =\frac{\gamma_{\min }}{\log \left(1+\frac{\Gamma_{m}^{p}}{\sigma^{2}}\right)}
\end{aligned}
$$

where $\omega_{m}{ }^{n}$ and $\omega_{m}{ }^{p}$ are the initial strategy vectors of FAPs and PBSs respectively. First of all the highly interfering MUEs to FAPs and PBSs are identified. Then each FAP and PBS maximize their utility function by exploring the favourable set of MUEs in each iteration giving the strategies of other FAPs and PBSs respectively. It is ensured in each iteration that a single MUE is not associated to multiple small base stations. It is also assured that before associating MUEs to FAPs and PBSs, after joining, combined bandwidth of FUEs and potential MUEs, and similarly PUEs and MUEs do not exceed the bandwidth allocated to FAPs and PBSs. Otherwise, the least interfering MUEs are dropped from the potential MUE joining list. Instead of trying each possible combination of MUE, to avoid complexity, greedy algorithm as in 21 can be used.

The next game is played by the MUEs to maximize their rates. The connected MUEs to FAPs, PBS and MBS probe the
TABLE 1 Simulation Parameters.

\begin{tabular}{|l|c|}
\hline Parameters & Value \\
\hline \hline BandWidth & $10 \mathrm{MHz}$ \\
\hline Macro eNodeBs & 1 \\
\hline Macro Cell Radius & $500 \mathrm{~m}$ \\
\hline Femto Cell Radius & $50 \mathrm{~m}$ \\
\hline Pico Cell Radius & $200 \mathrm{~m}$ \\
\hline Center Frequency & $2 \mathrm{GHz}$ \\
\hline UE transmit Power & $23 \mathrm{dBm}$ \\
\hline Indoor path-loss exponent $\beta$ & 2 \\
\hline Wall penetration loss $L$ & 0.5 \\
\hline Outdoor path-loss exponent $\alpha$ & 2.5 \\
\hline Noise variance $\sigma_{o}$ & $10^{-14}$ \\
\hline \hline
\end{tabular}

rates they are getting from their serving base stations in the previous game, MUEs stay connected if their utility is greater. The user centric scheme is distributed in nature, therefore it requires less computational and monitoring complexity. The algorithm in detail is explained in Algorithm 1.

\section{4 | PERFORMANCE EVALUATION}

In order to simulate the multi-tier HetNet scenario with and without network/infrastructure failure in order to evaluate the system performance in terms of the achievable sum rate subject to the coverage probability threshold and minimum QoS requirement constraints. The network snapshot is simulated in MATLAB by randomly putting some lower powered small cells such as PBS and FAP into the failure state ${ }^{2}$ In the simulated network/infrastructure failure snapshot, the maximum number of users that can be served by the multi-tier HetNet is assumed to be 20. For an illustrative purpose, the traffic load of $100 \%$ correspond to the 10 FAPs with each having 1 FUE and 2 PBSs with each having 2 PUEs lying within the geographical coverage of the MBS having 6 MUE $\$^{3}$ The simulation is carried out for two different type of experiments, i.e., in the first experimen $4^{4}$ the network snapshot is simulated to measure the performance of the proposed "Optimized User-Centric Scheme" in contrast to the traditional "All-Closed Scheme" without the network/infrastructure failure by using the following network setting such as 4 PBSs with each having 4 PUEs, 2 to 16 FAPs with each having 2 FUEs and 10 MUEs per MBS. In the second experiment, the simulations are carried out to

\footnotetext{
${ }^{2}$ It is important to highlight that the term network/infrastructure failure used throughout the paper pinpoints the complete failure meaning that the network access point cannot serve any user.

${ }^{3}$ It should be noted that this network setting is valid for the performance evaluation results depicted in the Figures 5-9.

${ }^{4}$ This network setting is valid for the Figures 3 and 4 .
} 


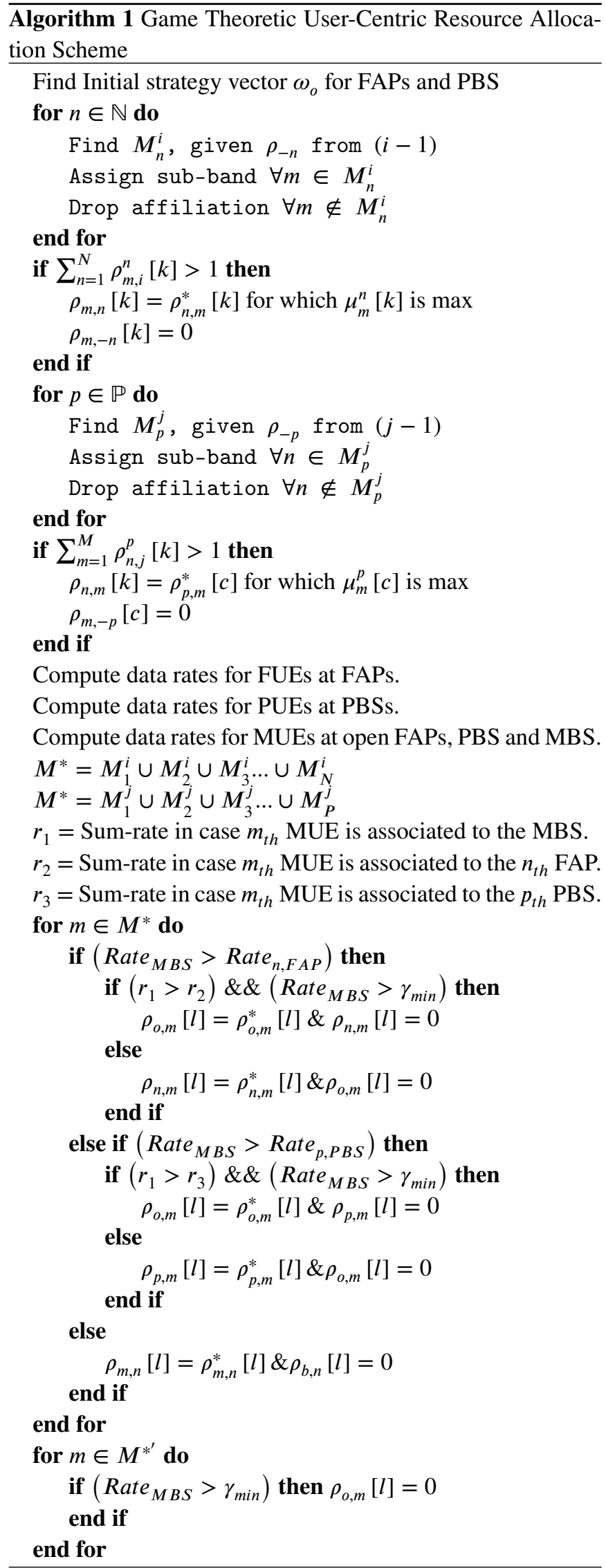

measure the optimized system sum-rate, outage probability/coverage probability for the different quality of service (QoS) requirements with the variable predicted average traffic load extracted from historical data, with and without varying infrastructure/network failure rates. The simulation parameters ${ }^{222}$ are shown in the Table 1.

Figure 3 depicts the relationship between the sum rate of the system against the number of FAPs while keeping the number of PBSs fixed to 4 . We consider a traffic load profile with the network setting such as as 4 PBSs with each having 4 PUEs, 2 to 16 FAPs with each having 2 FUEs and 10 MUEs per MBS wherein all the users are distributed randomly in the considered MBS of radius $500 \mathrm{~m}$ and the number of FUEs increases with an increase in the number of FAPs. The minimum traffic load consists of the total number of users to be 30 and the maximum traffic load corresponds to the total number of users to be 58. The two different access schemes are considered where in an all-closed access scheme, the FAPs cannot admit any nonhome users and the entire FAP bandwidth is dedicated to its home users. Similarly in the optimized user-centric scheme, the proposed formulated approach is considered wherein the users can decide which type of BS to connect with, based on the proposed user association procedure catering the interference mitigation as described in the aforementioned sections. It can be seen that the sum rate of the system decreases as the number of FAPs increases. This is due to the fact that the additional FAPs using the same spectrum causes more interference which reduces the achievable data rates. However, the proposed user-centric approach outperforms the all-closed scheme because of the interference management in the proposed user association procedure. It is seen that optimized sum rate is $56 \%$ better at minimum traffic load and $33 \%$ better at maximum traffic.

Figure 4 shows the comparison of outage probability between all-closed scheme and proposed optimized usercentric scheme with minimum QoS requirement set to 250 Kbps. It is clearly seen that in all-closed scheme, the outage probability is very high. This is due to the fact that randomly deployed MUEs in a large macro cell area experience higher path loss and uplink cross tier interference from FUEs and PUEs. As number of FAPs is increased the accumulated interference level further degrades the coverage of MUEs. Whereas in case of proposed optimized scheme outage probability is reduced to significant margin. PBSs and FAPs play open and MUEs can associate to either BS maximizing their data rates and releasing the bandwidth for the rest of MUEs thereby increasing overall data rates and resulting in reduced outage probability. As seen from the result that compared to all-closed scheme, the outage is reduced to $86 \%$ at minimum load and $75 \%$ at maximum traffic load. 
Figure 5 shows the relationship between coverage probability and traffic load at different quality of service requirement. It can clearly be seen that at least traffic load the coverage probability is higher, but as the traffic load increases, because of cross tier interference, the coverage also decreases. Furthermore as the QoS requirement increases, because of decrease in MUE data rate, the coverage probability also drops. Coverage probability achieved through proposed optimized scheme at traffic load varies from $25 \%$ to $100 \%$ is in the range from $95 \%$ to $85 \%, 90 \%$ to $79 \%, 88 \%$ to $74 \%$ and $83 \%$ to $70 \%$ with $250 \mathrm{Kbps}, 500 \mathrm{Kbps}, 750 \mathrm{Kbps}$ and $1 \mathrm{Mbps}$ QoS requirement, respectively.

The network resilience is investigated in terms of outage probability/coverage probability with $20 \%, 40 \%, 60 \%$ network/infrastructure failure rate at each service QoS requirements of $250 \mathrm{Kbps}, 500 \mathrm{Kbps}, 750 \mathrm{Kbps}, 1 \mathrm{Mbps}$. It can be clearly observed from Figure 6 and 7 that as the failure rate increases, outage probability increases. Furthermore at fixed failure rate an outage probability progressively increases with an increase in the traffic load. At the higher network/infrastructure failure rates more low powered base stations undergo a complete failure state which is chosen randomly and their users will become un-served, therefore due to path loss and fading outage probability of macro users is increased. Moreover when QoS requirement increases, because of not able to satisfy the data rate requirement, more users will go into outage. Figure 6 shows that in the proposed optimized user-centric scheme at $250 \mathrm{Kbps}$ QoS, minimum increase, in outage probability from without failure is $1.3 \%$ at $25 \%$ traffic load and $20 \%$ failure while the maximum increase in outage probability from without failure is $15 \%$ at $100 \%$ traffic load and $60 \%$ failure.

Similarly, the results shown in Figure 7 illustrates that in the proposed optimized scheme at $500 \mathrm{kbps}$ QoS, minimum increase, in outage probability from without failure is $4.2 \%$ at $25 \%$ traffic load and $20 \%$ failure while the maximum increase in outage probability from without failure is $14.5 \%$ at $100 \%$ traffic load and $60 \%$ failure.

The results shown in Figure 8 depict the relationship between coverage probability and traffic load at $750 \mathrm{Kbps}$ QoS requirement with/without BS failure rate. To meet higher QoS requirement, MUEs will go into low coverage, specially when failure rate increases. It is seen that in proposed optimized scheme, minimum decrease in coverage probability from without failure is approximately $0.5 \%$ at $25 \%$ traffic load and $20 \%$ failure while the maximum decrease in coverage probability from without failure is approximately $12 \%$ at $100 \%$ traffic load and $60 \%$ failure.

The results in Figure 9 show coverage probability at $1 \mathrm{Mbps}$ QoS requirement with/without BS failure. It is examined that at this higher QoS requirement our proposed scheme outperformed from $750 \mathrm{Kbps}$ QoS requirement at full load and 60\% failure. It can be clearly seen that from Figure 9 minimum decrease in coverage probability is approximately $2 \%$ at $25 \%$ traffic load and $20 \%$ failure while the maximum decrease in coverage probability is approximately $10 \%$ at $100 \%$ traffic load and $60 \%$ failure.

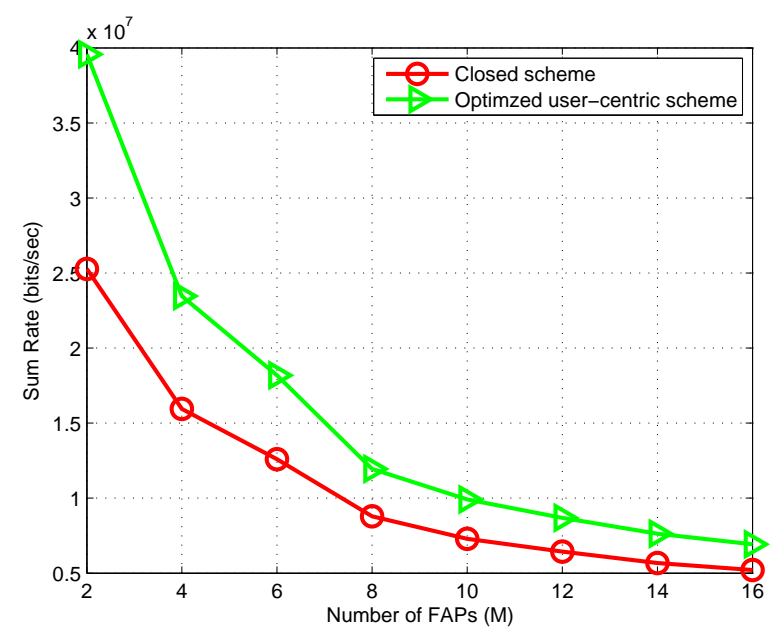

FIGURE 3 Effect of Varying number of FAPs on the System Sum Rate for All-Closed and Optimized User-Centric Schemes.

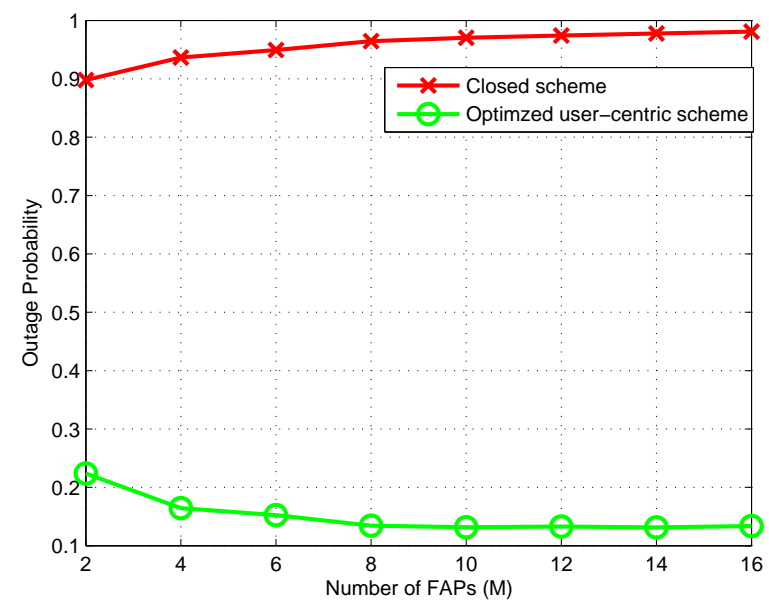

FIGURE 4 Outage Probability versus Number of FAPs. 


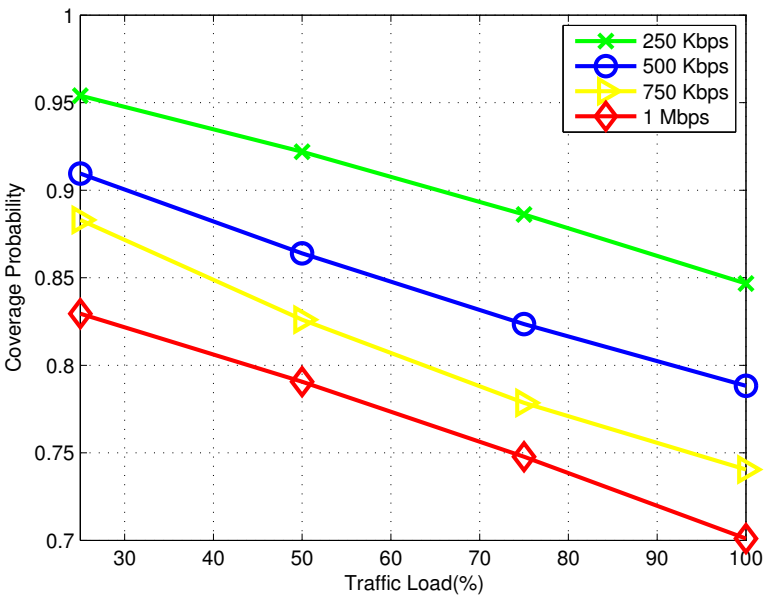

FIGURE 5 Coverage probability versus variable traffic load at different minimum QoS requirements.

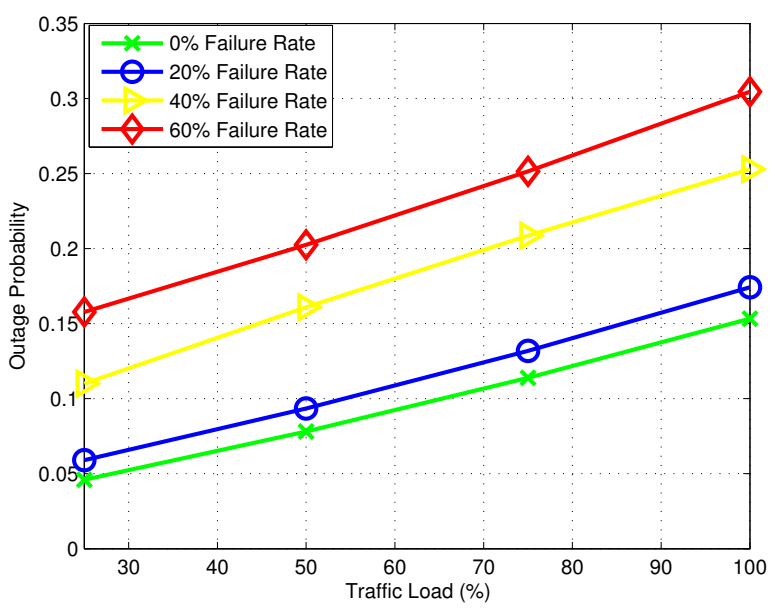

FIGURE 6 Outage probability versus variable traffic load for different network failure rates at minimum QoS requirement of $250 \mathrm{Kbps}$.

\section{5 | CONCLUSION}

In this investigation, the aim was to assess the $5 \mathrm{G}$ multi-tier network resilience in disaster situations. we have proposed non cooperative game theory based user-centric resource optimization scheme. The Nash equilibrium has been obtained as the solution for this game. we have shown in our proposed scheme that the Network resilience, quantified by the coverage probability, is enhanced by a significant margin as compared to all-closed scheme. The simulation results have shown that proposed scheme outperforms even at higher QoS requirements. We have analyzed the performance of the system in single

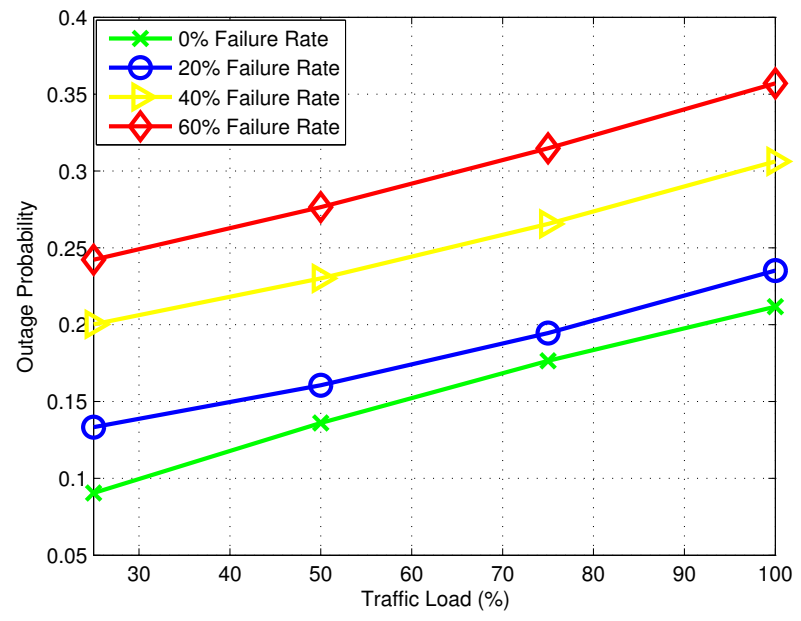

FIGURE 7 Outage probability versus variable traffic load for different network failure rates at minimum QoS requirement of $500 \mathrm{Kbps}$.

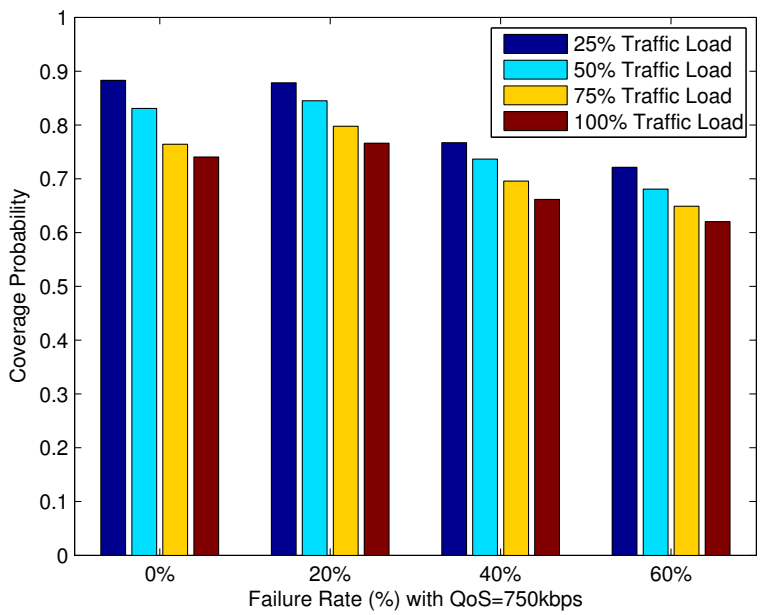

FIGURE 8 Coverage probability versus variable failure rate for different network traffic load at minimum QoS requirement of $750 \mathrm{Kbps}$.

macro cell scenario, in future the work can be extended to multicell scenario.

\section{References}

1. Mölleryd Bengt G, Markendahl Jan, Werding Jan, Mäkitalo Osten. Decoupling of revenues and traffic-Is there a revenue gap for mobile broadband?. 9th IEEE Conference on Telecommunications Internet and Media Techno Economics (CTTE). 2010;:1-7. 


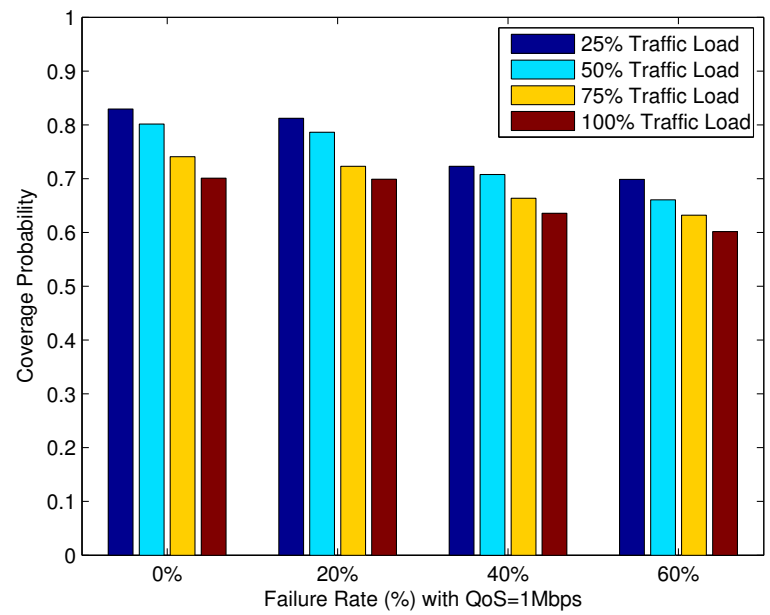

FIGURE 9 Coverage probability versus failure rate for different network traffic load at minimum QoS requirement of 1 Mbps.

2. Pervaiz Haris, Musavian Leila, Ni Qiang, Ding Zhiguo. Energy and spectrum efficient transmission techniques under QoS constraints toward green heterogeneous networks. IEEE Access. 2015;3:1655-1671.

3. Mudassir Ahmad, Akhtar Saleem, Kamel Hesham, Javed Abbas. Intelligent spectral efficiency and energy efficiency enhancement in LTE-Advanced heterogeneous networks. Transactions on Emerging Telecommunications Technologies. ;:e3431.

4. Yang Chungang, Li Jiandong, Ni Qiang, Anpalagan Alagan, Guizani Mohsen. Interference-aware energy efficiency maximization in 5G ultra-dense networks. IEEE Transactions on Communications. 2017;65(2):728-739.

5. Elsherif Ahmed R, Chen Wei-Peng, Ito Akira, Ding Zhi. Resource allocation and inter-cell interference management for dual-access small cells. IEEE Journal on Selected Areas in Communications. 2015;33(6):10821096.

6. Cheung Wang Chi, Quek Tony QS, Kountouris Marios. Throughput optimization, spectrum allocation, and access control in two-tier femtocell networks. IEEE Journal on Selected Areas in Communications. 2012;30(3):561-574.

7. Oo Thant Zin, Tran Nguyen H, Saad Walid, Niyato Dusit, Han Zhu, Hong Choong Seon. Offloading in HetNet: A Coordination of Interference Mitigation, User Association, and Resource Allocation. IEEE Transactions on Mobile Computing. 2017;16(8):2276-2291.
8. Andrews Jeffrey G, Singh Sarabjot, Ye Qiaoyang, Lin Xingqin, Dhillon Harpreet S. An overview of load balancing in HetNets: Old myths and open problems. IEEE Wireless Communications. 2014;21(2):18-25.

9. Wang Tao, Vandendorpe Luc. Iterative resource allocation for maximizing weighted sum min-rate in downlink cellular OFDMA systems. IEEE Transactions on Signal Processing. 2011;59(1):223-234.

10. Andrews Jeffrey G, Claussen Holger, Dohler Mischa, Rangan Sundeep, Reed Mark C. Femtocells: Past, present, and future. IEEE Journal on Selected Areas in communications. 2012;30(3):497-508.

11. Bennis Mehdi, Perlaza Samir M, Blasco Pol, Han Zhu, Poor H Vincent. Self-organization in small cell networks: A reinforcement learning approach. IEEE transactions on wireless communications. 2013;12(7):3202-3212.

12. Marden Jason R, Shamma Jeff S. Revisiting loglinear learning: Asynchrony, completeness and payoffbased implementation. Games and Economic Behavior. 2012;75(2):788-808.

13. Niyato Dusit, Hossain Ekram. Dynamics of network selection in heterogeneous wireless networks: An evolutionary game approach. IEEE transactions on vehicular technology. 2009;58(4):2008-2017.

14. Pantisano Francesco, Bennis Mehdi, Saad Walid, Debbah Merouane, Latva-Aho Matti. Interference alignment for cooperative femtocell networks: A game-theoretic approach. IEEE Transactions on Mobile Computing. 2013;12(11):2233-2246.

15. Bayat Siavash, Louie Raymond HY, Han Zhu, Vucetic Branka, Li Yonghui. Distributed user association and femtocell allocation in heterogeneous wireless networks. IEEE Transactions on Communications. 2014;62(8):3027-3043.

16. Zhang Zengfeng, Song Lingyang, Han Zhu, Saad Walid. Coalitional games with overlapping coalitions for interference management in small cell networks. IEEE Transactions on Wireless Communications. 2014;13(5):26592669.

17. Ni Qiang, Zarakovitis Charilaos C. Nash bargaining game theoretic scheduling for joint channel and power allocation in cognitive radio systems. IEEE Journal on selected areas in Communications. 2012;30(1):70-81.

18. Griffith David, Rouil Richard, Izquierdo Antonio, Golmie Nada. Measuring the resiliency of cellular base station 
deployments. IEEE Wireless Communications and Networking Conference (WCNC). 2015;:1625-1630.

19. Peng Chunyi, Lee Suk-Bok, Lu Songwu, Luo Haiyun, Li Hewu. Traffic-driven power saving in operational 3G cellular networks. Proceedings of the 17th annual international conference on Mobile computing and networking. 2011;:121-132.

20. Zhang Sheng, Zhao Shenglin, Yuan Mingxuan, et al. Traffic Prediction Based Power Saving in Cellular Networks: A Machine Learning Method. Proceedings of the 25th ACM SIGSPATIAL International Conference on Advances in Geographic Information Systems. 2017;:29.

21. Xia Ping, Chandrasekhar Vikram, Andrews Jeffrey G. Open vs. closed access femtocells in the uplink. IEEE Transactions on Wireless Communications. 2010;9(12):3798-3809.

22. Munir Hamnah, Hassan Syed Ali, Pervaiz Haris Bin, Ni Qiang, Musavian Leila. Energy efficient resource allocation in 5G hybrid heterogeneous networks: A game theoretic approach. 84th IEEE Vehicular Technology Conference, VTC Fall. 2016;:1-5. 